\title{
Emerging treatments in the management of bipolar disorder - focus on risperidone long acting injection
}

This article was published in the following Dove Press journal:

Neuropsychiatric Disease and Treatment

15 July 2010

Number of times this article has been viewed

\author{
Wissam El-Hage' \\ Simon A Surguladze ${ }^{2}$ \\ Inserm U930 ERL CNRS 3106 , \\ Université François Rabelais and \\ Clinique Psychiatrique Universitaire, \\ CHRU de Tours, Tours, France; \\ ${ }^{2}$ Institute of Psychiatry, King's College \\ London, UK
}

\begin{abstract}
Bipolar disorder is a life-long psychiatric illness characterized by a high frequency of relapses and substantial societal costs. Almost half of the patients are prescribed second generation antipsychotics for treatment of manic states, or as the maintenance therapy. Risperidone long acting injection (RLAI) as a monotherapy or as adjunctive therapy to lithium or valproate for the maintenance treatment of bipolar I disorder was approved by Food and Drug Administration (FDA) in United States in May 2009. In this review we will consider the aspects of pharmacology, pharmacokinetics, metabolism, safety and tolerability, and clinical trials focusing on the efficacy of RLAI in bipolar disorder. The patients' perspective and attitudes to long-acting injections will also be discussed.
\end{abstract}

Keywords: second generation, antipsychotics, patient attitudes, lithium, valproate, monotherapy

\section{Introduction}

Bipolar disorder (BD) is a major psychiatric disorder which for years has been underdiagnosed and - as a consequence - mistreated. The lifetime prevalence figures of BD based on studies conducted in 1990s eg, the Epidemiologic Catchment Area ${ }^{1}$ and National Comorbidity Survey ${ }^{2}$ study were $1.3 \%-1.6 \%$, respectively. More recent data gathered by National Comorbidity Survey-Replication (NCR-R) are based on a broader definition of BD spectrum and show that lifetime prevalence estimates are $4.5 \%$ for the BD spectrum, with 1.0\% for bipolar I disorder (BP-I), $1.1 \%$ for bipolar II disorder (BP-II), and 2.4\% for subthreshold BPD. ${ }^{3}$ BPD spectrum was defined as a lifetime history of BP-I, BP-II, or subthreshold BPD. Respondents were classified as having lifetime BP-I if they had ever had a manic episode; and as having lifetime BP-II if they had ever had a hypomanic, but not manic, episode and had ever had a major depressive episode (MDE). Definition of subthreshold BPD was based on any of the following: 1. recurrent subthreshold hypomania in the presence of intercurrent MDE, 2. recurrent hypomania in the absence of recurrent MDE, with or without subthreshold MDE, and 3. recurrent subthreshold hypomania in the absence of intercurrent MDE with, or without subthreshold MDE.

The economic costs of BD are substantial, which is related to a debilitating chronicity and high frequency of relapses in BD. The costs are estimated at $\$ 45$ billion a year in the United States (1991 values), with the economic losses due to work impairment accounting for the largest proportion of this total (nearly $\$ 18$ billion annually). ${ }^{4}$ In comparison the economic costs of major depression from a societal perspective are $\$ 53$ billion a year in the United States (1996 values). ${ }^{5}$
Correspondence: SA Surguladze Box PO69, Institute of Psychiatry, King's College London, DeCrespigny Park, London SE5 8AF, UK

Tel +442078480363

Fax +442078480572

Email simon.2.surguladze@kcl.ac.uk 
Subsequently, the need for the effective maintenance therapy of BD could not be overestimated. Although the main focus of this review is on the role of risperidone long acting injections (RLAI) in maintenance treatment of BD, we will first briefly consider existing practices in the use of oral risperidone and other second generation antipsychotics (SGA).

National Institute for Health and Clinical Excellence of the UK (NICE) guidelines ${ }^{6}$ recommend starting an antipsychotic, valproate or lithium when managing patients in acute manic or mixed episode. According to these guidelines, the second generation antipsychotics are preferred to first-generation antipsychotics. The SGAs currently licensed in the UK for the treatment of acute mania are olanzapine, risperidone and quetiapine.

A major advantage of the atypical antipsychotics over conventional antipsychotics is the lower risk of extrapyramidal symptoms (EPS), though this differential has largely been demonstrated in trials where the comparator is haloperidol, a high-potency conventional antipsychotic that is associated with a relatively high incidence of EPS.

As a long-term maintenance treatment, lithium and olanzapine have UK marketing authorization for the prophylaxis of bipolar disorder, and carbamazepine has marketing authorization for the prophylaxis of bipolar disorder unresponsive to lithium.

In the USA all SGAs (except clozapine) have received the FDA indication for BD. Apart from use in acute conditions, SGAs appear to be of a broad use at any stage of BD. Thus, Sajatovic $^{7}$ in a report based on the US Veterans health registry, demonstrated that $45 \%(n=32,994)$ of patients with BD were on antipsychotics as monotherapy or combination therapy. The great majority of the individuals $(77.5 \%, \mathrm{n}=25,559)$ prescribed antipsychotic agents received SGAs.

\section{Current treatment options Risperidone}

The studies show that among other SGAs, oral risperidone may play an important role in long-term treatment of bipolar disorder. For example, a large-scale multicenter open-label study involving adjunctive risperidone treatment of 541 patients with bipolar disorder or schizoaffective dis$\operatorname{order}^{8}$ demonstrated significant, sustained improvements in manic and depressive symptoms as well as Clinical Global Impression scores. The open, adjunctive nature of risperidone use constitutes the major limitation of this study.

There have been concerns about the long-term use of risperidone with regard to its potential to induce EPS. A naturalistic, retrospective study comparing clozapine, risperidone, and olanzapine found no significant difference in efficacy or occurrence of EPS among the three groups. ${ }^{9}$

Another study of risperidone ${ }^{10}$ looked at both the shortterm and continuation therapy of bipolar mania. This was a randomized, double-blind trial of 438 patients, 154 of whom were randomized to risperidone, 144 to haloperidol, and 140 to placebo. After the initial phase of 3 weeks, the patients were followed up to another 9 weeks where the effects of haloperidol and risperidone were compared. The authors report that risperidone monotherapy was an effective and well-tolerated treatment for bipolar mania and that efficacy was maintained over the long term.

\section{Aripiprazole}

Aripiprazole (a partial D2 agonist, 5HT2A antagonist and 5HT1A partial agonist) was evaluated in a 100-week double-blind, placebo-controlled, relapse prevention study. ${ }^{11}$ Efficacy and tolerability of aripiprazole appeared superior to placebo in delaying time to manic relapse; however, no significant differences were observed in time to depressive relapse.

Aripiprazole has also showed good efficacy in a small (28 patient) study of patients with rapid-cycling BD which demonstrated that aripiprazole was generally well tolerated in the long-term treatment (100 weeks) of rapid-cycling bipolar disorder. ${ }^{12}$

\section{Quetiapine}

Quetiapine in adjunction with lithium/divalproex has been found to be an effective long-term treatment option for bipolar I disorder in preventing recurrences not only of mania but also depression; for example Vieta at $\mathrm{a}^{13}$ examined the efficacy and safety of quetiapine in combination with lithium or divalproex as compared with placebo and lithium or divalproex, and found that treatment with quetiapine in combination with lithium/divalproex significantly increased time to recurrence of any event (mania, depression, or mixed) irrespective of the polarity of the index episode compared with placebo. Long-term treatment with quetiapine was generally well tolerated.

In another trial, ${ }^{14}$ after the 12 week period of open-label stabilization, 628 patients were randomly assigned to doubleblind treatment with quetiapine or placebo, in combination with lithium or divalproex, for up to 104 weeks. The primary efficacy measure was time to recurrence of any mood event (mania, depression, or a mixed episode). The results showed that fewer patients in the quetiapine group experienced a mood event compared with the placebo group (20.3\% versus $52.1 \%$ ). 
The beneficial effect of quetiapine in relapse prevention was demonstrated in a small (21-patient) adolescent sample as well. ${ }^{15}$

Seroquel XR (quetiapine fumarate) Extended-Release Tablets were approved in 2008 by the FDA for the maintenance treatment of BP-I as an adjunct therapy to lithium or divalproex. It also has FDA approval as monotherapy for treatment for bipolar disorder-acute depressive episodes, and bipolar I disorder-acute manic or mixed episodes.

\section{Olanzapine}

Olanzapine has been used most widely for the long-term treatment of $\mathrm{BD}$ and there is a large amount of data on its efficacy and tolerability. The recent Cochrane review ${ }^{16}$ evaluated the effects of olanzapine as a monotherapy or adjunctive treatment in preventing manic, depressive, and mixed episodes in patients with bipolar affective disorder, based on 5 trials with 1165 participants.

The analysis showed that there was no statistically significant difference between olanzapine and placebo (either alone or in combination with lithium or valproate) in terms of number of participants who experienced relapse into mood episode; however restricting the analysis to the trial that compared olanzapine monotherapy versus placebo, there was a statistically significant difference in favor of olanzapine. No statistically significant difference was found between olanzapine and other mood stabilizers (lithium or valproate) in preventing symptomatic relapse for any mood episode, however, olanzapine was more effective than lithium in preventing symptomatic manic relapse. As a downside, olanzapine either alone or as adjunctive treatment to mood stabilizers was associated with significantly greater weight gain than placebo. Although olanzapine was associated with a lower rate of manic relapse, there was evidence for higher rate of weight increase and depression associated with olanzapine than lithium. The authors concluded that the evidence is in favor of lithium as the first line maintenance treatment of bipolar disorder.

\section{Monotherapy versus combined therapy}

The decision - which medication to choose for the maintenance treatment and whether or not administer it as a monotherapy or combined with antimanic medication - is not a trivial one. This is particularly important when considering the maintenance treatment with a long-acting medication, eg, RLAI.

First, it is essential to consider that the profiles of responders to lithium, valproate and SGAs are different. The author of a thoughtful review ${ }^{17}$ gives examples where, for example, manic patients who had previously responded well to lithium later responded well to lithium again, but not to divalproex. Similarly, it has been reported that olanzapine treatment succeeded in patients who had previously failed on lithium and divalproex treatment. The author concludes that these "observations of selective response do not support the clinical impression that many bipolar patients benefit partially from several different treatments and that, therefore, these treatments usually need to be combined". Subsequently, because monotherapies can often be helpful in carefully selected bipolar patients, the author recommends the use of combinations in more specific situations - for example, when a patient fails on adequate monotherapy, when clinical data provide no leads as to which monotherapy to choose (such as in an early episode), or when a patient does not tolerate adequate monotherapy.

\section{Compliance problems}

One of the problems encountered by the prescribers of longterm treatment of $\mathrm{BD}$ is the patients' poor compliance with the medication.

The factors associated with medication compliance could be grouped to those related to patient's personality, effects of illness, or effects of the medication. A recent study ${ }^{18}$ noted that the range of treatment non-adherence in patients with $\mathrm{BD}$ varies from $20 \%-70 \%$ in different studies. The results of that study showed that $12 \%$ were largely nonadherent to long-term treatment. The authors concluded that non-adherent patients with bipolar disorder received less intensive pharmacologic treatments, had more suicide attempts, and experienced more barriers to care than adherent individuals.

There have been indications that the treatment adherence depends on the type of medication. For example, the study ${ }^{19}$ showed that the patients treated with quetiapine had significantly greater regularity of prescription refills than individuals treated with typical antipsychotics, risperidone, or olanzapine. Quetiapine and risperidone were associated with a longer treatment duration than the typical antipsychotics, olanzapine, or ziprasidone. The authors suggest that the clinicians should aim at achieving the optimum balance between efficacy and adverse effects.

In some non-compliant patients with bipolar disorder who would benefit from a long-term antipsychotic, a long-acting injection (LAI) may aid compliance. Although not specifically addressing $\mathrm{BD}$, a recent editorial ${ }^{20}$ provides very useful overview of advantages of the LAI over the oral formulations and vice versa. For example, easier and early detection of 
relapse, relapse prevention, and reduced re-hospitalization rates could count as the definite advantages of LAI, whereas the oral formulations may prove advantageous with regard to the possibility of rapid discontinuation in cases of serious side effects, providing patients with an enhanced sense of autonomy and reducing stigma.

There are only two SGAs available as the long-acting injections: olanzapine pamoate and risperidone long acting injections (RLAI).

Olanzapine pamoate is licensed in the UK for longterm treatment of schizophrenia, but there have been safety concerns due to post-injection delirium/sedation syndrome (PDSS). ${ }^{21}$ This requires a risk management plan that includes supervision of the patient by health care personnel for 3 hours post-injection. To date there have been no published articles on use of this preparation in BD.

\section{The use of risperidone long-acting injections \\ Introduction to the compound}

The active component of RLAI - risperidone - is a benzisoxazole derivative with potent combined serotonin 5HT2A and dopamine D2 receptor-blocking properties. Adrenergic $\alpha 1$ and to a lesser extent, histamine $\mathrm{H} 1$ and adrenergic $\alpha 2$ receptors are also antagonized by risperidone.

To date there have been several studies, and one review article, ${ }^{22}$ addressing the role of RLAI in the maintenance treatment of BD.

RLAI as monotherapy, or as adjunctive therapy to lithium or valproate, for the maintenance treatment of BP-I was approved by FDA in United States in May 2009. The recommended dose for monotherapy, or adjunctive therapy to lithium or valproate, for the maintenance treatment of BP-I is $25 \mathrm{mg}$ IM every 2 weeks. Some patients may benefit from a higher dose of $37.5 \mathrm{mg}$ or $50 \mathrm{mg}$. Dosages above $50 \mathrm{mg}$ have not been studied in this population.

In the UK long-acting intramuscular injections of antipsychotics ('depots') are not recommended for routine use in bipolar disorder. NICE recommends though that they may be considered for people who were treated successfully for mania with oral antipsychotics, but have had a relapse because of poor adherence.

\section{Pharmacology, pharmacokinetics and neuroimaging data on RLAI}

Typical antipsychotic depot preparations are delivered in an oil-based suspension, with a high level of side-effects related to the oil-based medium in which the medication is suspended. Particularly, this can lead to the development of nodules at the injection site over time. RLAI is a waterbased suspension that contains the active drug in glycolic acid-lactate microspheres. As the parent compound risperidone lacks the hydroxyl group traditionally used to bind to an ester to facilitate the production of a slow-release mechanism, a delivery system of glycolic acid-lactate copolymer microspheres containing the drug was developed. Following injection, the microspheres are progressively hydrolyzed, leading to a slow and steady release of risperidone over a period of several weeks. The majority of the release occurs during weeks 4-6 after the injection. Therefore the patients should be maintained on oral risperidone for the first three weeks of initiation of RLAI.

The pharmacokinetic profile of RLAI exhibits less peakto-trough variability than that of oral risperidone, and lower peak plasma concentrations, as a result of the delayed release of active agent from the vehicle microspheres. ${ }^{23}$ After injection, less than $1 \%$ of the administered dose is released initially; the main release of risperidone occurs after a period of 3 weeks. Release is sustained during weeks 4 to 6 and begins to subside by week 7 . The elimination phase is complete at 7 to 8 weeks following microsphere breakdown. Repeated administration of RLAI every 2 weeks achieves steady-state plasma levels after the fourth injection. Therefore, the use of RLAI ensures continuous antipsychotic coverage. ${ }^{24}$ The pharmacokinetics of RLAI are linear in the usual dose range of 25 to $50 \mathrm{mg}$.

Oral risperidone undergoes significant 'first-pass' metabolism $-90 \%$ is bound in plasma to albumin and alpha-1 acid glycoprotein prior to elimination via the kidneys. Plasma levels are normal in patients with hepatic insufficiency, but may be raised by $60 \%$ in patients with renal impairment. The advantages of RLAI formulation include the avoidance of first-pass metabolism, attenuated fluctuations between peak and trough concentrations, and the certainty of medication delivery. Eerdekens et $\mathrm{al}^{24}$ evaluated the pharmacokinetics and tolerability of RLAI in a multicenter, prospective, openlabel, 15-week study of 86 patients with schizophrenia. They found that the mean steady-state peak concentrations of the active moiety were significantly lower with intramuscular than oral dosing, and fluctuations in plasma active-moiety levels were significantly lower with the RLAI than oral risperidone.

Nesvåg et $\mathrm{al}^{25}$ showed that the patients receiving RLAI had lower mean concentration of risperidone plus 9-OHrisperidone, and a lower 9-OH-risperidone/risperidone ratio 
than patients taking oral risperidone. The difference in mean total serum concentration was mainly due to lower mean concentration of 9-OH-risperidone in the group receiving RLAI, while the mean concentration of risperidone was equal in the two groups. The lower level of 9-OH-risperidone in the RLAI group may represent reduced first-pass metabolism.

The pharmacological activity of risperidone and 9-OH-risperidone is claimed to be similar, but no direct evidence for this has been reported. It has been proposed that the drug's therapeutic activity is mediated through a combination of dopamine Type 2 (D2) and serotonin Type 2 (5HT2) receptor antagonism.

Positron emission tomography studies have shown a central dopamine receptor occupancy of $70 \%$ to be associated with clinical efficacy for typical oral antipsychotic agents. A D2-occupancy of 53\%-85\% was found in seven patients treated with a daily dose of 3-6 mg of oral risperidone. ${ }^{26}$ Another positron emission tomography study showed a D2-occupancy of 25\%-72\% in eight patients after receiving three or five injections of 25-75 mg RLAI. ${ }^{27}$

Functional magnetic resonance (fMRI) studies of patents with schizophrenia demonstrated that treatment with RLAI - as compared with the first generation depot antipsychotics - was associated with a normalisation of blood oxygenation level dependent (BOLD) response in the medial prefrontal cortex in tasks involving working memory ${ }^{28}$ and facial emotion processing. ${ }^{29}$

Following subcutaneous injections of risperidone, 9-OH-risperidone was found to have a limited distribution compared with risperidone in frontal and striatal regions in the rat brain. ${ }^{30}$ These differences in distribution of risperidone and 9-OH-risperidone in the rat brain indicate that the parent drug and metabolite may have different pharmacokinetic properties which may be relevant to the antipsychotic efficacy of the agent. ${ }^{31}$ Intramuscular injections of depot antipsychotic agents imply a more stable serum drug level and improved compliance compared with oral medication. ${ }^{32}$ Thus a lower mean serum drug level prior to next injection may not necessarily imply lower antipsychotic efficacy throughout the time span between injections. However, among patients receiving oral risperidone there is only a weak association between serum drug levels and clinical response and adverse effects. ${ }^{33}$ This is also the case for typical depot agents. Therefore, possible clinical implications of a difference in serum drug levels between oral and RLAI remain obscure.

\section{Administration}

Thyssen et $\mathrm{al}^{34}$ reported the results of two studies in chronic schizophrenic individuals where RLAI was administered into the deltoid versus gluteal muscle. The first study included a large sample of 170 patients in a multicenter, open-label, single-dose, 2-way, cross-over design. During each treatment period, patients received a single injection into the deltoid or gluteal muscle on day 1 followed by an 85-day observation/ washout period. In the second study, they reported the safety and tolerability of RLAI administered into the deltoid. This was an 8-week open-label, multicenter, multidose study with 53 patients who required higher doses and who had previously received RLAI gluteal injections. During the 8-week treatment phase, patients received 4 sequential intramuscular injections of RLAI $37.5 \mathrm{mg}$ or $50 \mathrm{mg}$, administered every 2 weeks into the deltoid muscle. In both studies, RLAI doses were administered with $2 \mathrm{~mL}$ of diluent as an intramuscular injection. The $2 \mathrm{~mL}$ diluent volume was considered as an acceptable volume for deltoid injections of aqueous fluids, and is the approved marketed volume for gluteal injections of RLAI . This multidose study was designed to evaluate the discontinuation rate of schizophrenic subjects receiving multiple biweekly deltoid injections of 37.5 or $50 \mathrm{mg}$ of RLAI. This study had an $83 \%$ completion rate. In both studies, no patient withdrew due to injection-site tolerability issues.

In the single-dose study, there was slightly higher incidence of injection site reactions following deltoid injection compared with gluteal injection. However, these reactions were mild and did not appear to be clinically significant. Furthermore, none of the subjects who discontinued did so because of reasons related to the injection site. Investigators rated the injection site reactions which did not reveal any nodule formations. In the majority of patients, postadministration pain was rated as either minimal or none in the first 24 hours following injection.

The shape of the plasma concentration-time profiles following single deltoid and gluteal injection were similar. The shape of plasma concentration-time curves of the active moiety, risperidone, and 9-OH-risperidone were similar in the different treatment groups. Peak active moiety plasma concentrations were achieved after approximately 30 days for all treatments, with an apparent elimination half-life of 6 to 8 days, independent of dose or injection site. Bioequivalence was demonstrated irrespective of the pre-dose concentration of the active moiety. The plasma exposure increased with dose, and dose-proportionality was shown after deltoid injection of $37.5 \mathrm{mg}$ and $50 \mathrm{mg}$ of RLAI and after gluteal injection of $25 \mathrm{mg}$ and $50 \mathrm{mg}$ of RLAI. Cross-panel and cross-study graphical 
comparison for the different deltoid and gluteal treatments showed that dose-normalized pharmacokinetic parameters were similar across treatments (ie, doses and injection sites).

Thus, based on the pharmacokinetic results, the authors concluded that RLAI injection was safe and well tolerated when administered as a single gluteal injection or as a single deltoid injection or multiple deltoid injections. The injection of RLAI into the deltoid muscle can offer patients a more acceptable and convenient route of administration that may enhance overall long-term treatment adherence.

\section{Safety and tolerability issues}

Malempati et $\mathrm{al}^{35}$ found that the RLAI was well tolerated in all patients with bipolar disorder. EPS were present in 30\% of the bipolar patients and were reduced compared with prior medication therapy in $30 \%$ of the patients. Only one patient required concomitant treatment with an anticholinergic medication. The average weight gain over 2 years was low $(1.6 \mathrm{~kg})$ and other side effects were minimal.

The studies below report on various side effects of RLAI treatment in mixed groups of patients. Han et $\mathrm{al}^{36}$ found no significant body weight change in their study during RLAI treatment, although 4 participants switched to RLAI due to weight gain. On the other hand, Mohl et $\mathrm{al}^{37}$ reported a small but significant body weight shift in their study using RLAI in stable patients with schizoaffective disorder.

In a retrospective study, Camacho et $\mathrm{al}^{38}$ enrolled 50 patients suffering from schizophrenia or bipolar disorder with a history of poor medication adherence. The results revealed generally high patient adherence with such a program and meaningful improvements in attendance to their appointments as well as global functioning. In terms of their weight, it was noted that patients receiving RLAI for at least one year had $3 \%$ increase of their mean weight. These results are only observational since patients were receiving other psychotropic drugs in addition to the RLAI.

In the study of Niaz and Haddad, ${ }^{39}$ the sample comprised 90 patients with schizophrenia, schizoaffective, or bipolar affective disorder. No adverse effects of RLAI treatment were present in one third of the patients. One third had no record of the presence or absence of adverse effects, and the last one third recorded an adverse effect attributed to RLAI. The most common adverse effect was a movement disorder (EPS including akathisia), but also a raised prolactin, increased sedation, sexual dysfunction, hypersalivation, weight gain, painful injection, anxiety and sleep disturbance. In this sample, half of the 90 patients who commenced RLAI had stopped it by the evaluation point. Lack of efficacy accounted for approximately $40 \%$ and intolerability for approximately $20 \%$. Despite these results, this study showed that RLAI was associated with a reduction in total admissions, compulsory admissions, and total inpatient days and was cost-effective.

When reviewing the results of side effects of various LAI across different studies, Taylor ${ }^{40}$ pointed to huge inconsistencies in dosage, with the likelihood that LAI-treated patients may often be significantly overdosed. This consideration should be taken into account when evaluating the side effects of RLAI as well.

\section{Use of RLAl as monotherapy and as an adjuvant to lithium or valproate}

Only a small number of studies have been published on the use of RLAI in bipolar disorder. The patient samples in some studies have been mixed - eg, comprising individuals with bipolar disorder and schizophrenia or schizoaffective disorder.

In an open label observational study with RLAI, ${ }^{41}$ 14 patients met the diagnostic criteria of BP-I and 8 patients met the diagnostic criteria of schizophrenia according to the DSM-IV-TR. These patients were characterized by the treatment non-compliance and frequent relapses that led to the subsequent hospitalization. The maintenance treatment was followed up to 40 weeks during which no depressive symptoms or significant adverse effects were observed.

Ten outpatients with BP-I or BP-II were prescribed openlabel RLAI 25-50 mg every 2 weeks as an adjunct to ongoing treatment with mood stabilizing and other medications. ${ }^{35}$ Depressive episodes predominated over manic/hypomanic/ mixed episodes in all patients prior to initiation of treatment with RLAI. During the 2-year follow-up period, manic symptoms declined over time in all patients and depressive symptoms were minimal. No patients required hospitalization for mood episodes. One patient suffered a manic episode, one experienced hypomania, and two had mixed episodes. All mood elevations were relatively mild and all patients demonstrated improvement in psychosocial functioning during the follow-up period. The authors concluded that RLAI may be useful in poorly compliant bipolar patients at high risk of manic relapse.

On a small sample of 11 bipolar patients, ${ }^{36}$ the authors showed that RLAI may be beneficial in the maintenance therapy of stable bipolar patients. In this exploratory trial, the maintenance of a euthymic mood state was achieved during the 12 months of treatment, with low Young Mania Rating Scale (YMRS) scores that could be regarded to indi- 
cate remission. Moreover, the patients and the caregivers reported high levels of satisfaction. The treatment was well tolerated and showed advantages in achieving compliance. The authors recommend the use of small doses of RLAI (25 mg), which would be a positive aspect for long-term successful management of BP-I.

Yatham et $\mathrm{al}^{42}$ prospectively assessed the safety and efficacy of RLAI in comparison to oral atypical antipsychotics (risperidone, quetiapine, or olanzapine) in the longer term treatment of bipolar disorder, using a 6-month, open-label, randomized design. The 49 bipolar outpatients who were treated with a mood stabilizer and an oral second-generation antipsychotic at baseline were randomized to either continue their respective antipsychotic regimen or to switch to RLAI.

In RLAI patients, and those on oral atypical antipsychotic patients, there were similar rates of at least one treatmentrelated adverse event ( $70 \%$ and $73 \%$, respectively). Most frequently reported adverse events for RLAI were insomnia, nausea, fatigue and headache. Also in this study, RLAI was commenced at $25 \mathrm{mg}$ for a minimum of 6 weeks, with stringent recommendations regarding dose increases. Each subsequent RLAI dose increase was maintained for a minimum of 4 weeks before permitting a final dose increase. The authors argued that this limitation may have biased the results in favor of oral atypical antipsychotic. Still, this study showed that RLAI has similar efficacy and tolerability in comparison to oral atypical antipsychotics.

A 6-month case series of 12 bipolar patients ${ }^{43}$ reported a response rate of $100 \%$ by endpoint, with no depressive episodes in any patients. RLAI was well tolerated, somatic complaints were limited, with $8 \%$ reporting dizziness and $16 \%$ reporting constipation. No significant adverse events (including EPS) occurred. RLAI significantly reduced the severity of symptoms, and no manic or depressive episodes were seen in these previously noncompliant patients in 6-month period. Also, switching from mood stabilizers, or mood stabilizers plus atypical antipsychotics, to RLAI, or adding on RLAI, was safe and well tolerated in our population. These naturalistic, uncontrolled observations suggest the potential of RLAI in the long-term management of BP-I patients.

In the first prospective study to assess the effectiveness of RLAI in a cohort of bipolar patients, Vieta et $\mathrm{al}^{44}$ entered 29 acutely manic bipolar inpatients in a mirror-design, observational study. They received naturalistic treatment for a manic episode plus RLAI for a mean period of 2 years. Side effects were reported by nine patients. Five of them reported EPS and needed antiparkinsonian medication. Three patients had prolactin level elevation and one complained of impotence. Another patient reported depression as a side effect. Only four subjects stopped the treatment due to the side effects. Weight gain occurred in five patients (17\%). Weight gain was found to be similar to the weight gained during oral risperidone therapy.

The only double-blind, randomized trial published to date was supported by Ortho-McNeil Janssen Scientific Affairs. ${ }^{45}$ This study included patients with BD-I with four and more mood episodes in the 12 months prior to study entry. Following a 16-week, open-label stabilization phase with RLAI plus treatment as usual (TAU), remitted patients entered a 52-week, double-blind, placebo-controlled, relapseprevention phase. Randomized patients continued treatment with adjunctive RLAI (25-50 mg every 2 weeks) plus TAU $(n=65)$ or switched to adjunctive placebo injection plus TAU $(n=59)$. The primary outcome measure was time to relapse to any mood episode. Out of 240 enrolled patients, 124 entered double-blind treatment. Time to relapse was longer in patients receiving adjunctive RLAI $(P=0.010)$. Relative relapse risk was 2.3-fold higher with adjunctive placebo $(P=0.011)$. Side effect profile was more benign in adjunctive RLAI group compared with that in adjunctive placebo one. The authors concluded that adjunctive RLAI significantly delayed time to relapse in patients with BP-I who relapse frequently.

\section{Efficacy studies in elderly patients}

Bipolar disorder in older adult populations has gained increasing attention due to the growing proportion of elderly, eg, in the US it is predicted to increase from about 4 million in 1970 to 15 million in $2030 .^{46}$

A study of Hirshfeld et $\mathrm{al}^{47}$ reported the prevalence of bipolar disorder at $0.5 \%$ among individuals aged 65 and older as bipolar illness persists into later life.

A continuing unmet need is the identification of agents that are generally well tolerated and effective in later-life bipolar disorder. Medications that are first-line treatments in younger patients, such as lithium, may be poorly tolerated in older patients due to side effects and renal dysfunction. Additional strategies for treatment of bipolar disorder in late-life are needed.

No data is available concerning the efficacy of RLAI in elderly patients with BD.

The choice of an antipsychotic medication should be based on patients' history of response and personal preference, clinician's previous experience, and pharmacokinetic properties. 
Caution is required in older patients with renal or hepatic impairment, and in subjects with decreased rates of renal blood flow, glomerular filtration, and renal clearance, as well as reduced levels of synthesis and activity of hepatic microsomal enzymes. An age-related fall in total body water results in higher plasma concentrations which may increase both activity and the potential for adverse effects of RLAI as water-based compound. ${ }^{48}$

A report of RLAI treatment of 52 elderly patients (65 years and older) with schizophrenia, schizoaffective, and other psychotic disorders may serve as an approximate guide. ${ }^{49}$ The patients were switched from stable antipsychotic regimens. The most common dosage of RLAI used at endpoint was $25 \mathrm{mg}$ every 14 days. Conversion to RLAI resulted in significant improvements in movement disorder severity, psychiatric symptoms, functional status. and patient satisfaction. In general, the results showed that RLAI was well tolerated and safe in elderly patients with psychotic illnesses.

\section{Patient-focused perspectives such as quality of life, patient satisfaction, acceptability, adherence and uptake}

There is little data available regarding long-term use of RLAI in patients with BD. The question remains to be answered - will the patients stay on this medication once stabilized? We can only provide an example from the study of schizophrenia patients ${ }^{50}$ where the investigators found that out of 211 patients, over 3 years, $84 \%$ of subjects discontinued RLAI; $27.7 \%$ of these switched to oral risperidone. Amongst the factors predicting greater probability of discontinuation were younger age, longer duration of illness, inpatient status at initiation, and an RLAI dose of $25 \mathrm{mg} / 2$ weeks.

The cross-sectional study ${ }^{51}$ sheds light on other factors that could be relevant for RLAI treatment in BD. In particular, these authors evaluated perceived treatment effects, patients concerns, and expectations among 90 individuals with BD. The study showed that while individuals with BD appreciated the effects of medications on keeping them stable, their concerns regarding adverse effects were not insignificant. Another aspect, relevant for long-term treatment, was the perceived discrepancy between actual and hoped-for outcome.

Thus, the question arises - what is it that the clinician is offering to patient "in exchange" to regular visits to the depot clinic? Is it a better quality of life? This latter aspect deserves further investigation.

\section{Conclusion}

The efficacy of RLAI in maintenance treatment of BD has been evaluated in a small number of studies, with one published report of a double-blind, randomized controlled trial thus far. ${ }^{45}$ Much has to be done in terms of establishing the true value of this preparation in the long-term management of $\mathrm{BD}$.

The studies published to date converge on highlighting the positive effects of RLAI in terms of improving adherence, reducing the relapse rates, and overall good tolerability.

Still, there are more questions to be answered regarding the place that RLAI might have in the long-term therapy of BD. In particular how cost-effective is the long-term use of RLAI? Do the patients need the RLAI at all in adjunction to the well established antimanic agents ie, lithium, valproate or carbamazepine? Why don't we aim for monotherapy? And, if we do, could RLAI prove effective as a monotherapy in maintenance treatment of BD? How can we justify the longterm use of injectable antipsychotic to BD patients - eg, when they are in remission? Shall we tell them that BD is a kind of chronic psychosis?

A recent editorial in The British Journal of Psychiatry dedicated to the role of LAI in the treatment of schizophrenia ${ }^{52}$ emphasized that it was a "very real issue that most people are uncomfortable about the prospect of taking long-acting drugs particularly drugs that can profoundly change how one feels, with no way of reversing these effects for several weeks". This might be particularly true in the case of BD where patients feel "back to normal" after recovering from the mood episode.

More studies directly addressing these questions are warranted, as well as a broader discussion related to the patient's perspective with regard to long-term injectable antipsychotics in general.

\section{Acknowledgment}

The authors would like to thank Dr MX Patel for her helpful comments on the draft of this paper.

\section{Disclosures}

SAS has been reimbursed for the attendance at scientific conferences by Janssen-Cilag and Sanofi-Syntelabo. He alsohas received speaker fees from AstraZeneca and Eli Lilly within the past 5 years.

WEH has received speaker fees from BMS, Eli Lilly, Janssen-Cilag and Lundbeck. He received research grantsfrom Servier, the French Association of Biological Psychiatry (AFPB) and Lundbeck, which are unrelated to the content of this manuscript. 


\section{References}

1. Weissman MM, Bruce L, Leaf PJ, Florio L, Holzer C. Affective Disorders. In: Robins LN, Regier DA, editors. Psychiatric Disorders in America. The Epidemiologic Catchment Area Study. New York: The Free Press; 1991. p. 53-80.

2. Kessler R. The National Comorbidity Survey of the United States. Int Rev Psychiatry. 1994;6:365-376.

3. Merikangas KR, Akiskal HS, Angst J, et al. Lifetime and 12-month prevalence of bipolar spectrum disorder in the National Comorbidity Survey replication. Arch Gen Psychiatry. 2007 May;64(5): 543-552.

4. Kleinman L, Lowin A, Flood E, Gandhi G, Edgell E, Revicki D. Costs of bipolar disorder. Pharmacoeconomics. 2003;21(9): 601-622.

5. Greenberg PE, Kessler RC, Birnbaum HG, et al. The economic burden of depression in the United States: how did it change between 1990 and 2000? J Clin Psychiatry. 2003 Dec;64(12):1465-1475.

6. National Institute for Health and Clinical Excellence. Bipolar Disorder. The Management of Bipolar Disorder in Adults, Children and Adolescents, in Primary and Secondary Care. National Collaborating Centre for Mental Health ed. The British Psychological Society and The Royal College of Psychiatrists; 2006.

7. Sajatovic M, Madhusoodanan S, Fuller MA, Aulakh L, Keaton DB. Risperidone for bipolar disorders. Expert Rev Neurother. 2005 Mar; 5(2):177-187.

8. Vieta E, Goikolea JM, Corbella B, et al. Risperidone safety and efficacy in the treatment of bipolar and schizoaffective disorders: results from a 6-month, multicenter, open study. J Clin Psychiatry. 2001 Oct;62(10): $818-825$.

9. Guille C, Sachs GS, Ghaemi SN. A naturalistic comparison of clozapine, risperidone, and olanzapine in the treatment of bipolar disorder. J Clin Psychiatry. 2000 Sep;61(9):638-642.

10. Smulevich AB, Khanna S, Eerdekens M, Karcher K, Kramer M, Grossman F. Acute and continuation risperidone monotherapy in bipolar mania: a 3-week placebo-controlled trial followed by a 9-week doubleblind trial of risperidone and haloperidol. Eur Neuropsychopharmacol. 2005 Jan;15(1):75-84.

11. Keck PE Jr, Calabrese JR, McIntyre RS, et al. Aripiprazole monotherapy for maintenance therapy in bipolar I disorder: a 100-week, double-blind study versus placebo. J Clin Psychiatry. 2007 Oct;68(10): 1480-1491.

12. Muzina DJ, Momah C, Eudicone JM, et al. Aripiprazole monotherapy in patients with rapid-cycling bipolar I disorder: an analysis from a long-term, double-blind, placebo-controlled study. Int J Clin Pract. 2008 May;62(5):679-687.

13. Vieta E, Suppes T, Eggens I, Persson I, Paulsson B, Brecher M. Efficacy and safety of quetiapine in combination with lithium or divalproex for maintenance of patients with bipolar I disorder (international trial 126). J Affect Disord. 2008 Aug;109(3): 251-263.

14. Suppes T, Vieta E, Liu S, Brecher M, Paulsson B. Maintenance treatment for patients with bipolar I disorder: results from a north american study of quetiapine in combination with lithium or divalproex (trial 127). Am J Psychiatry. 2009 Apr;166(4):476-488.

15. Duffy A, Milin R, Grof P. Maintenance treatment of adolescent bipolar disorder: open study of the effectiveness and tolerability of quetiapine. BMC Psychiatry. 2009;9:4.

16. Cipriani A, Rendell JM, Geddes J. Olanzapine in long-term treatment for bipolar disorder. Cochrane Database Syst Rev. 2009;(1): CD004367.

17. Grof P. Selecting effective long-term treatment for bipolar patients: monotherapy and combinations. J Clin Psychiatry. 2003;64 Suppl 5: 53-61.

18. Sajatovic M, Biswas K, Kilbourne AK, Fenn H, Williford W, Bauer MS. Factors associated with prospective long-term treatment adherence among individuals with bipolar disorder. Psychiatr Serv. 2008 Jul; 59(7):753-759.
19. Gianfrancesco FD, Rajagopalan K, Sajatovic M, Wang RH. Treatment adherence among patients with bipolar or manic disorder taking atypical and typical antipsychotics. J Clin Psychiatry. 2006 Feb;67(2): 222-232.

20. Patel MX, Taylor M, David AS. Antipsychotic long-acting injections: mind the gap. Br J Psychiatry. 2009 Nov 1;195(52):S1-S4.

21. Citrome L. Olanzapine pamoate: a stick in time? A review of the efficacy and safety profile of a new depot formulation of a second-generation antipsychotic. Int J Clin Pract. 2009 Jan;63(1):140-150.

22. Kemp DE, Canan F, Goldstein BI, McIntyre RS. Long-acting risperidone: a review of its role in the treatment of bipolar disorder. Adv Ther. 2009 Jun;26(6):588-599.

23. Ereshefsky L, Mascarenas CA. Comparison of the effects of different routes of antipsychotic administration on pharmacokinetics and pharmacodynamics. J Clin Psychiatry. 2003;64 Suppl 16:18-23.

24. Eerdekens M, Van HI, Remmerie B, Mannaert E. Pharmacokinetics and tolerability of long-acting risperidone in schizophrenia. Schizophr Res. 2004 Sep 1;70(1):91-100.

25. Nesvag R, Hendset M, Refsum H, Tanum L. Serum concentrations of risperidone and 9-OH risperidone following intramuscular injection of long-acting risperidone compared with oral risperidone medication. Acta Psychiatr Scand. 2006 Jul;114(1):21-26.

26. Nyberg S, Eriksson B, Oxenstierna G, Halldin C, Farde L. Suggested Minimal Effective Dose of Risperidone Based on PET-Measured D2 and 5-HT2A Receptor Occupancy in Schizophrenic Patients. Am J Psychiatry. 1999 Jun 1;156(6):869-875.

27. Gefvert O, Eriksson B, Persson P, et al. Pharmacokinetics and D2 receptor occupancy of long-acting injectable risperidone (Risperdal Consta) in patients with schizophrenia. Int J Neuropsychopharmacol. 2005 Mar;8(1):27-36.

28. Surguladze SA, Chu EM, Evans A, et al. The effect of long-acting risperidone on working memory in schizophrenia: a functional magnetic resonance imaging study. J Clin Psychopharmacol. 2007 Dec; 27(6):560-570.

29. Surguladze SA, Chu EM, Marshall N, et al. Emotion processing in schizophrenia: fMRI study of patients treated with risperidone long-acting injections or conventional depot medication. Journal of Psychopharmacology. 2010; in press.

30. van Beijsterveldt LE, Geerts RJ, Leysen JE, et al. Regional brain distribution of risperidone and its active metabolite 9-hydroxyrisperidone in the rat. Psychopharmacology (Berl). 1994 Feb;114(1): 53-62.

31. Aravagiri M, Marder SR. Brain, plasma and tissue pharmacokinetics of risperidone and 9-hydroxyrisperidone after separate oral administration to rats. Psychopharmacology (Berl). 2002 Feb;159(4):424-431.

32. Kane JM, Aguglia E, Altamura AC, et al. Guidelines for depot antipsychotic treatment in schizophrenia. European Neuropsychopharmacology Consensus Conference in Siena, Italy. Eur Neuropsychopharmacol. 1998 Feb;8(1):55-66.

33. Riedel M, Schwarz MJ, Strassnig M, et al. Risperidone plasma levels, clinical response and side-effects. Eur Arch Psychiatry Clin Neurosci. 2005 Aug;255(4):261-268.

34. Thyssen A, Rusch S, Herben V, Quiroz J, Mannaert E. Risperidone Long-Acting Injection: Pharmacokinetics Following Administration in Deltoid Versus Gluteal Muscle in Schizophrenic Patients. J Clin Pharmacol. 2010 Jan 23.

35. Malempati RN, Bond DJ, Yatham LN. Depot risperidone in the outpatient management of bipolar disorder: a 2-year study of 10 patients. Int Clin Psychopharmacol. 2008 Mar;23(2):88-94.

36. Han C, Lee MS, Pae CU, Ko YH, Patkar AA, Jung IK. Usefulness of long-acting injectable risperidone during 12-month maintenance therapy of bipolar disorder. Progress in NeuroPsychopharmacology and Biological Psychiatry. 2007;31: 1219-1223.

37. Mohl A, Westlye K, Opjordsmoen S, et al. Long-acting risperidone in stable patients with schizoaffective disorder. J Psychopharmacol. 2005 Sep;19 Suppl 5:22-31. 
38. Camacho A, Ng B, Galangue B, Feifel D. Use of risperidone long-acting injectable in a rural border community clinic in southern california. Psychiatry (Edgmont). 2008 Jun;5(6):43-49.

39. Niaz OS, Haddad PM. Thirty-five months experience of risperidone long-acting injection in a UK psychiatric service including a mirrorimage analysis of in-patient care. Acta Psychiatr Scand. 2007 Jul; 116(1):36-46.

40. Taylor D. Psychopharmacology and adverse effects of antipsychotic long-acting injections: a review. Br J Psychiatry. 2009 Nov 1;195(52): S13-S19.

41. Benabarre A, Castro P, Sanchez-Moreno J, et al. Efficacy and safety of long-acting injectable risperidone in maintenance phase of bipolar and schizoaffective disorder]. Actas Esp Psiquiatr. 2009 May;37(3): 143-147.

42. Yatham LN, Fallu A, Binder CE. A 6-month randomized open-label comparison of continuation of oral atypical antipsychotic therapy or switch to long acting injectable risperidone in patients with bipolar disorder. Acta Psychiatr Scand Suppl. 2007;(434):50-56.

43. Savas HA, Yumru M, Ozen ME. Use of long-acting risperidone in the treatment of bipolar patients. J Clin Psychopharmacol. 2006 Oct; 26(5):530-531.

44. Vieta E, Nieto E, Autet A, et al. A long-term prospective study on the outcome of bipolar patients treated with long-acting injectable risperidone. World J Biol Psychiatry. 2008;9(3):219-224.

45. Macfadden W, Alphs L, Haskins JT, et al. A randomized, doubleblind, placebo-controlled study of maintenance treatment with adjunctive risperidone long-acting therapy in patients with bipolar I disorder who relapse frequently. Bipolar Disord. 2009 Dec;11(8): $827-839$.
46. Jeste DV, Alexopoulos GS, Bartels SJ, et al. Consensus statement on the upcoming crisis in geriatric mental health: research agenda for the next 2 decades. Arch Gen Psychiatry. 1999 Sep;56(9):848-853.

47. Hirschfeld RM, Calabrese JR, Weissman MM, et al. Screening for bipolar disorder in the community. J Clin Psychiatry. 2003 Jan;64(1): 53-59.

48. Singh D, O'Connor DW. Efficacy and safety of risperidone long-acting injection in elderly people with schizophrenia. Clin Interv Aging. 2009; 4:351-355.

49. Kissling W, Glue P, Medori R, Simpson S. Long-term safety and efficacy of long-acting risperidone in elderly psychotic patients. Hum Psychopharmacol. 2007 Dec;22(8):505-513.

50. Taylor DM, Fischetti C, Sparshatt A, Thomas A, Bishara D, Cornelius V. Risperidone long-acting injection: a prospective 3-year analysis of its use in clinical practice. J Clin Psychiatry. 2009 Feb;70(2): 196-200.

51. Sajatovic M, Jenkins JH, Cassidy KA, Muzina DJ. Medication treatment perceptions, concerns and expectations among depressed individuals with Type I Bipolar Disorder. J Affect Disord. 2009 Jun;115(3):360-366

52. Burns T. Knowledge about antipsychotic long-acting injections: bridging that gap. Br J Psychiatry. 2009 Nov 1;195(52):S5-S6.
Neuropsychiatric Disease and Treatment

\section{Publish your work in this journal}

Neuropsychiatric Disease and Treatment is an international, peerreviewed journal of clinical therapeutics and pharmacology focusing on concise rapid reporting of clinical or pre-clinical studies on a range of neuropsychiatric and neurological disorders. This journal is indexed on PubMed Central, the 'PsycINFO' database and CAS, and is the official

\section{Dovepress}

journal of The International Neuropsychiatric Association (INA). The manuscript management system is completely online and includes a very quick and fair peer-review system, which is all easy to use. Visit http://www.dovepress.com/testimonials.php to read real quotes from published authors. 